\title{
(6) OPEN ACCESS \\ Social disparities in Disease Management Programmes for coronary heart disease in Germany: a cross-classified multilevel analysis
}

\author{
Kayvan Bozorgmehr, ${ }^{1}$ Werner Maier, ${ }^{2}$ Hermann Brenner, ${ }^{3}$ Kai-Uwe Saum, ${ }^{3}$ \\ Christian Stock, ${ }^{4}$ Antje Miksch, ${ }^{1}$ Bernd Holleczek, ${ }^{5}$ Joachim Szecsenyi, ${ }^{1}$ Oliver Razum ${ }^{6}$
}

\begin{abstract}
- Additional material is published online only. To view please visit the journal online (http://dx.doi.org/10.1136/jech2014-204506)
\end{abstract}

For numbered affiliations see end of article.

\section{Correspondence to} Dr Kayvan Bozorgmehr, Department of General Practice and Health Services Research, University Hospital Heidelberg, Voßstr.2, Geb. 37, Heidelberg 69115, Germany; kayvan.bozorgmehr@med. uni-heidelberg.de

Received 10 June 2014 Revised 24 February 2015 Accepted 19 May 2015 Published Online First 16 June 2015

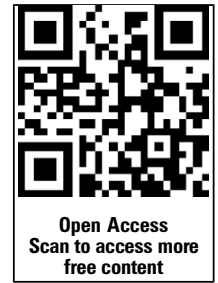

\section{CrossMark}

To cite: Bozorgmehr $\mathrm{K}$ Maier W, Brenner $H_{\text {, et al. J }}$ Epidemiol Community Health 2015:69:1091-1101.

\section{ABSTRACT}

Background Disease Management Programmes (DMPs) aim to improve effectiveness and equity of care but may suffer from selective enrolment. We analysed social disparities in DMP enrolment among elderly patients with coronary heart disease (CHD) in Germany, taking into account contextual effects at municipality and primary care practice levels.

Methods Cross-sectional analysis of effects of educational attainment and regional deprivation on physician-reported DMP enrolment in a subsample of a large population-based cohort study in Germany, adjusting for individual-level, practice-level and area-level variables. We calculated OR and their 95\% Cls $(95 \% \mathrm{Cl})$ in cross-classified, multilevel logistic regression models. Results Among $\mathrm{N}=1280$ individuals with CHD (37.3\% women), DMP enrolment rates were $22.2 \%$ (women) and 35\% (men). The odds of DMP enrolment were significantly higher for male patients $(\mathrm{OR}=1.98$ (1.50 to 2.62)), even after adjustment for potential confounding by individual-level, practice-level and area-level variables (range: $\mathrm{OR}=1.60$ (1.08 to 2.36 ) to 2.16 (1.57 to 2.98$)$ ). Educational attainment was not significantly associated with DMP enrolment. Compared to patients living in least-deprived municipalities, the adjusted propensity of DMP enrolment was statistically significantly lower for patients living in medium-deprived municipalities $(\mathrm{OR}=0.41(0.24$ to 0.71$))$, and it also tended to be lower for patients living in the most-deprived municipalities $(\mathrm{OR}=0.70(0.40$ to 1.21$))$. Models controlling for the social situation (instead of healthrelated behaviour) yielded comparable effect estimates (medium-deprived/most-deprived vs least-deprived areas: $\mathrm{OR}=0.45(0.26$ to 0.78$) / \mathrm{OR}=0.68(0.33$ to 1.19$)$ ).

Controlling for differences in comorbidity attenuated the deprivation effect estimates.

Conclusions We found evidence for marked gender, but not educational disparities in DMP enrolment among patients with CHD. Small-area deprivation was associated with DMP enrolment, but the effects were partly explained by differences in comorbidity. Future studies on DMPs should consider contextual effects when analysing programme effectiveness or impacts on equity and efficiency.

\section{INTRODUCTION}

Coronary heart disease (CHD) is the leading cause of death and an important cause of morbidity worldwide $^{12}$ and in Germany. ${ }^{3}$ CHD is among the major chronic diseases covered by Disease Management Programmes (DMPs), which were introduced between 2002 and 2005 into the German statutory health insurance system (SHI). The rationale was to improve quality of care, ${ }^{4}$ provide financial incentives for purchasers and providers to care for the chronically ill ${ }^{5}$ and to improve survival and quality of life of enrolled patients. ${ }^{6}$ The first DMPs for CHD (DMP-CHD) were introduced in 2003. Ten years later, more than 1.7 million patients across Germany were enrolled in about 1700 accredited DMPs for CHD.

An unresolved issue concerning all DMPs is whether there is selective enrolment, favouring patients with higher socioeconomic status (SES). This would affect conclusions on the effectiveness of DMPs regarding the programme goals. ${ }^{8}{ }^{9}$ Three mechanisms, operating at different levels, could lead to disparities in DMP enrolment: First, participation in DMPs is voluntary for patients. Uptake might be inversely associated with need and individual SES. ${ }^{10}$ Second, physicians have the mandate to enrol only 'active patients' with respect to their therapy who can potentially benefit from the programme, ${ }^{6}$ which might be those who are already adherent to physician recommendations and have less risk factors, amplifying pre-existing behavioural barriers towards uptake of preventive programmes for patients with lower SES. Third, contextual factors of the small-area social environment ${ }^{11}$ such as neighbourhood socioeconomic disadvantage, ${ }^{12} 13$ as well as characteristics at the level of primary care practices,${ }^{14}$ might jointly or independently affect the access to and utilisation of DMPs.

The aim of this study was to assess social disparities in enrolment in DMPs among elderly people with CHD. We analysed whether individual educational attainment and regional deprivation are independently associated with DMP enrolment, considering potential confounding by characteristics at individual-level, practice-level and/or arealevel.

\section{METHODS}

Design, study population and context

This analysis was based on a subsample of the "Epidemiological Study for the Prevention, Early Diagnosis and Optimal Treatment of Chronic Diseases in an Elderly Population" (ESTHER), a prospective cohort study including non-institutionalised people from the general population living in the federal state of Saarland/Germany, who were recruited by their general practitioners (GPs) during a general health check-up between 2000 and 2002 $(\mathrm{t} 0)$. Baseline recruitment $(\mathrm{t} 0)$ occurred before DMPs were introduced. $^{5}$ 
A total of 9949 out of 12000 invited individuals, aged 5075 years, agreed to participate in the cohort. This sample is representative for the population of Saarland in the respective age range. ${ }^{15}$ Numerous GP-reported and patient-reported measures were captured by postal questionnaires at baseline and after two $(\mathrm{t} 1)$, five $(\mathrm{t} 2)$, eight $(\mathrm{t} 3)$ and $11(\mathrm{t} 4)$ years of follow-up. ${ }^{15-17}$

Saarland is divided into 52 municipalities (Gemeinden) comprising $1.2 \%$ of the overall population in Germany. Population sizes at the municipality level range from 6100 inhabitants (smallest) to more than 175800 (largest).

\section{Inclusion and exclusion criteria}

Since information on DMP enrolment was not available prior to t3 (2008-2010), we a priori excluded $\mathrm{N}=2937$ individuals. These were non-responders (ie, participants for whom neither patient questionnaires nor GP-reported information was available, $n=694)$, and participants who died $(n=1033)$ or dropped out $(n=1210)$ on health grounds $(n=253)$ or other and unknown reasons $(n=957)$ since $t 0$. At $t 3$, the response rate among survivors who were still physically and mentally able to participate was $80.9 \%(\mathrm{~N}=7012)$. Exclusion criteria specific to this study were defined as not having a GP-reported diagnosis of myocardial infarction (MI) and/or angina pectoris (AP) between t0 and $\mathrm{t} 3(\mathrm{n}=5694)$, missing data on baseline SES $(n=36)$ and being institutionalised at $\mathrm{t} 3(\mathrm{n}=2)$. A non-responder analysis was performed comparing the cohort population at $\mathrm{t} 3$ $(\mathrm{N}=7012)$ with those who were a priori excluded $(\mathrm{N}=2937)$ with respect to relevant baseline criteria (see online supplementary file 2).

\section{Outcome, exposure and covariables}

The outcome was the GP-reported enrolment status (yes/no) in the DMP-CHD at $\mathrm{t} 3$. Self-reported DMP-CHD status was used for $n=133$ individuals for whom GP-reported diagnosis of MI and/or AP, but no GP-reported information on DMP-CHD status, was available.

\section{Educational attainment}

The highest educational attainment at baseline was used to approximate individual-level SES. It was defined as an ordinal variable with three levels (table 1 ).

\section{Area-level SES}

We used the German Index of Multiple Deprivation (GIMD) for the year $2006^{18} 19$ to assess the effects of small-area deprivation on the propensity of DMP enrolment. Patients and their GPs were assigned a distinct value of regional deprivation at the municipality level by linking their ZIP codes (at $\mathrm{t} 3$ ) to one of 52 municipalities ('patient-residential area' and 'GP-residential area'). Municipalities were grouped into tertiles (table 1) in relation to all 9620 municipalities in Germany.

\section{Individual-level covariables}

As part of a variable selection process, we considered three categories of individual-level characteristics (beyond age and sex) as potential confounders in separate models. These were (table 1):

I. Comorbidities captured by the Cumulative Illness Rating Scale for Geriatrics (CIRS-G) severity index ${ }^{20}$ to adjust for differences in need;

II. Behavioural risk factors;

III. Ethnic differences in terms of immigration background and the social situation.

We present the results of the comorbidity-adjusted models since these are essential for analyses of disparities in healthcare. ${ }^{21}$ The other models are presented in online supplementary file 1 (tables S1-S2).

\section{Practice-level and area-level covariables}

We controlled for potential differences in DMP enrolment that could arise due to (1) provider characteristics ${ }^{14}$ (differences in gender of GPs that could lead to different treatment of individuals based on their social group characteristics such as educational attainment or deprivation of place of residence) or due to (2) rural/urban characteristics following the definition of the European Union Commission ${ }^{22}$ (table 1).

\section{Missing data}

Patients without ZIP codes $(n=14)$ or GP identifiers $(n=25)$ were not included in the regression analysis. Missing data in covariables were treated as missing at random and a complete case analysis was performed.

\section{Statistical analysis}

We calculated absolute frequencies and proportions stratified by sex for all variables used in this study. Owing to the cross-nested nature of the data, we assessed the effects of educational attainment and area-level SES on DMP enrolment using crossclassified, multilevel logistic regression models. In contrast to conventional multilevel analysis, cross-classified models consider the fact that patients are simultaneously nested both in their residential area and in GP practices, while patients' and GPs' residential areas may differ. In the cross-classified model, patients (level 1) are nested at level 2 in the cross-classification of 'GPs-by-municipality' (or more specifically: "GP-practicesby-patient-residential-area”).

We calculated the median ORs (MORs) ${ }^{23}$ to quantify the degree of clustering at higher levels, and tested the model fit of crossclassified null models by means of likelihood-ratio tests against three alternative models (see online supplementary file 1 ).

We present crude and adjusted subject-specific OR with their 95\% CIs to quantify the fixed effects of the predictors on the outcome. We calculated the proportional change in variance (PCV) at municipality-level and practice-level, respectively, in order to assess the effects of individual-level, practice-level and/ or area-level variables on the random part. ${ }^{24}$ To check for violations of the assumption of normality of random effects that may arise due to small average cluster sizes quantile-quantile plots were created (see online supplementary file 1 ). All analyses were performed using Stata V.12.1.

\section{RESULTS}

\section{Descriptive results}

The lifetime prevalence of physician-reported CHD in the cohort at $\mathrm{t} 3 \quad(\mathrm{~N}=7012)$ was $18.8 \% \quad(\mathrm{n}=1318)$. Of these, $\mathrm{N}=1280$ (37.3\% women) fulfilled all inclusion criteria. The mean age was 72.3 years (SD 6.2). Three-quarters of the subsample with CHD had a low educational attainment and $47.8 \%$ lived in municipalities categorised as most deprived (T3; table 2).

Absolute and relative differences in deprivation scores between the most and least deprived municipality were 25.50 index points and $327 \%$, respectively. The lifetime prevalence of physician-reported $\mathrm{MI}$ in the study population $(\mathrm{N}=1280)$ was lower among female participants $(26.3 \%)$ compared to male participants $(44.1 \%)$, while the prevalence of AP was fairly balanced. The vast majority $(72.6 \%)$ of patients enrolled in the DMP-CHD ( $\mathrm{N}=387)$ were male. The overall DMP enrolment 
Table 1 Definition of outcome, exposure and covariables

\begin{tabular}{|c|c|}
\hline & Definition \\
\hline \multicolumn{2}{|l|}{ Outcome } \\
\hline DMP enrolment & GP-reported enrolment status (yes/no) in the DMP-CHD at t3 (2008-2010) \\
\hline \multicolumn{2}{|l|}{ Exposure } \\
\hline \multicolumn{2}{|l|}{ Individual-level } \\
\hline Highest educational attainment & $\begin{array}{l}\text { Level I (lowest): no formal degree or at least } 9 \text { years of schooling qualifying for professional training, Hauptschule } \\
\text { Level II (medium): at least } 10 \text { years of schooling qualifying for professional training, Realschule/Mittlere Reife } \\
\text { Level III (highest): at least } 12 \text { or } 13 \text { years of schooling qualifying for university entrance, Fachhochschulreife/Abitur }\end{array}$ \\
\hline \multicolumn{2}{|l|}{ Area-level } \\
\hline $\begin{array}{l}\text { Regional deprivation of patients' residential areas } \\
\text { (GIMD) }\end{array}$ & $\begin{array}{l}\text { The GIMD includes seven domains of area deprivation: income, employment, education, municipal or district } \\
\text { revenue, social capital, environment and security. Absolute deprivation scores were grouped into tertiles comprising } \\
\text { T1: least-deprived municipalities } \\
\text { T2: medium-deprived municipalities } \\
\text { T3: most-deprived municipalities }\end{array}$ \\
\hline
\end{tabular}

Covariables

Comorbidity

Cumulative IIIness Rating Scale for Geriatrics

(CIRS-G) severity index

Behavioural risk factors

Hypercholesterolaemia

Smoking status

Alcohol consumption in g/day according to the WHO drinking categories*

GP-reported comorbidities calculated as CIRS-G score divided by the number of endorsed CIRS-G categories

Self-reported diagnosis of hypercholesterolaemia (yes/no) (ever diagnosed)

Self-reported smoking status in three categories 'Never smoker', 'Former smoker' and 'Current smoker'

Self-reported alcohol consumption of beer, wine or liquor per week

Abstainers: reporting a consumption of $0 \mathrm{~g} /$ day

DI: female: 0-19.99; male: 0-39.99

DII: female: 20-39.99; male: 40-59.99

DIII: female: $>40.0$; male: $>60.0$

Body mass index (BMI)

Self-reported weight (in kilograms) over height (to the power of two) in four categories

$\mathrm{BMl}<25$ : underweight-normal

BMI 25.00-29.99: Overweight/pre-obese

BMI 30.00-39.99: Obese class I+II

$\mathrm{BMI} \geq 40$ : Obese Class III

Social situation and ethnicity

Immigration background (yes/no)

Having an immigration background was defined as having (1) a foreign nationality or (2) a German nationality and a place of birth outside of Germany

Living in a partnership (yes/no)

Social contacts

Self-reported answer to question: do you live in a partnership?

The number of social contacts was included as a proxy of loneliness. It was captured by the question "How many family members/relatives/friends do you have which whom you can discuss any problems and on which you can rely?". The responses were collapsed into an ordinal variable with three categories ('0-1', '2-4' and '5-10 and more'

Approved need of long-term care

Need of long-term care as approved by the Medical Review Board of Statutory Health Insurance Funds (Medizinischer Dienst der Krankenkassen) as a self-report. The variable was used as a measure to approximate ability to participate in social life

Current economic activity

Self-reported current economic activity, categorical variable comprising six categories ('Full-time employment', 'Minor/occasional employment', 'Part-time employment', 'Housewife/Domestic activity', 'Retired' and 'Unemployed')

Area characteristics

Degree of urbanisation

Rural: $<300$ inhabitants $/ \mathrm{km}^{2}$ or less than 5000 inhabitants

Urban cluster: $\geq 300$ inhabitants $/ \mathrm{km}^{2}$ and minimum population of 5000 inhabitants, but less than 50000

High-density cluster: $\geq 1000$ inhabitants $/ \mathrm{km}^{2}$ and minimum population of 50000 inhabitants

\footnotetext{
All data refer to the 8-year follow-up phase (t3: 2008-2010) if not otherwise stated.

*Amount of alcohol per type of alcohol used to calculate categories: 1 bottle of beer=11.88 g; 1 glass of wine=22.0 g; 1 shot of liquor=6.4 g.

GP, general practitioner; DMP-CHD, Disease Management Programmes coronary heart disease; GIMD, German Index of Multiple Deprivation.
}

rates were $22.2 \%$ among women with $\mathrm{CHD}(\mathrm{N}=478)$ and $35 \%$ among men $(\mathrm{N}=802)$. More details are shown in table 2.

Out of all GP practices ( $\mathrm{N}=391)$, the majority $(76.6 \%)$ drew their patients with $\mathrm{CHD}$ from only one (not necessarily their own) municipality. The number of GP practices per municipality ranged from as few as one GP to 83 GPs. The average number of patients with CHD residing in the 52 municipalities or being treated in the 391 practices was 24.3 (SD: 33.8) and 3.2 (SD: 4.4), respectively. About 90\% $(n=1103)$ of the patients for whom linkage to both patient-residential area and GP-residential area was possible $(\mathrm{N}=1221)$ were treated by primary care physicians whose practices were located in municipalities with deprivation tertiles equal to that of their patients with CHD. Thus, the overall heterogeneity at practice level in terms of SES among patients with CHD was relatively low.

\section{Regression results}

Crude analysis

According to the crude models, the odds of enrolment in the DMP-CHD were significantly higher-regardless of the place of residence or practice-level effects-for male participants, participants categorised to drinking category DI compared to abstainers and patients with a higher severity of comorbidities (CIRS-G). The crude point estimates for the effects of educational attainment and GIMD tertiles on DMP enrolment 
Table 2 Descriptive characteristics of included participants of the ESTHER study with coronary heart disease at the 8-year follow-up $(2008-2010)(\mathrm{N}=1280)$

\begin{tabular}{|c|c|c|c|c|}
\hline & $\begin{array}{l}\text { Female } \\
\text { Freq. (col \%) }\end{array}$ & Male & Total & $\begin{array}{l}\text { Missing } \\
\text { Freq. ( } \% \text { of } N \text { ) }\end{array}$ \\
\hline \multicolumn{5}{|l|}{ Individual-level characteristics } \\
\hline \multicolumn{5}{|l|}{ Age group } \\
\hline 55-64 & $33(6.9)$ & $132(16.5)$ & $165(12.9)$ & \\
\hline $65-74$ & $241(50.4)$ & $386(48.1)$ & $627(49)$ & \\
\hline $75-84$ & $204(42.7)$ & $284(35.4)$ & $488(38.1)$ & \\
\hline N (\%) & $478(100)$ & $802(100)$ & $1280(100)$ & $0(0)$ \\
\hline \multicolumn{5}{|l|}{ Highest educational attainment* } \\
\hline Level I (lowest) & $402(84.1)$ & $591(73.7)$ & $993(77.6)$ & \\
\hline Level II & $56(11.7)$ & $84(10.5)$ & $140(10.9)$ & \\
\hline Level III (highest) & $20(4.2)$ & $127(15.8)$ & $147(11.5)$ & \\
\hline $\mathrm{N}(\%)$ & $478(100)$ & $802(100)$ & $1280(100)$ & $0(0)$ \\
\hline \multicolumn{5}{|l|}{ Immigration background } \\
\hline Yes & $45(9.5)$ & $60(7.5)$ & $105(8.2)$ & \\
\hline $\mathrm{N}(\%)$ & $475(100)$ & $798(100)$ & $1273(100)$ & $7(0.5)$ \\
\hline \multicolumn{5}{|c|}{ Lifetime prevalence of physician-reported CHD defining morbidities/index diseases } \\
\hline \multicolumn{5}{|l|}{ Myocardial infarction } \\
\hline Yes & $118(26.3)$ & $346(44.1)$ & $464(37.6)$ & \\
\hline $\mathrm{N}(\%)$ & $448(100)$ & $785(100)$ & $1233(100)$ & $42(3.3)$ \\
\hline \multicolumn{5}{|l|}{ Angina pectoris } \\
\hline Yes & $459(96)$ & $745(93.2)$ & $1204(94.3)$ & \\
\hline $\mathrm{N}(\%)$ & $478(100)$ & $799(100)$ & $1277(100)$ & $3(0.2)$ \\
\hline \multicolumn{5}{|l|}{ Comorbidities and behavioural risk factors } \\
\hline \multicolumn{5}{|l|}{ CIRS-G—severity index } \\
\hline $\mathrm{M}(\mathrm{SD})$ & $1.60(0.43)$ & $1.61(0.45)$ & $1.61(0.44)$ & \\
\hline $\mathrm{N}$ & 394 & 663 & 1057 & $223(17.4)$ \\
\hline \multicolumn{5}{|l|}{ Hypercholesterolaemia } \\
\hline Yes & $322(73.7)$ & $509(67.2)$ & $831(69.5)$ & \\
\hline $\mathrm{N}(\%)$ & $437(100)$ & $758(100)$ & $1195(100)$ & $85(6.6)$ \\
\hline \multicolumn{5}{|l|}{ Smoking status } \\
\hline Never smoker & $270(72.2)$ & $217(32)$ & $487(46.3)$ & \\
\hline Former smoker & $74(19.8)$ & $406(59.9)$ & $480(45.6)$ & \\
\hline Current smoker & $30(8)$ & $55(8.1)$ & $85(8.1)$ & \\
\hline $\mathrm{N}(\%)$ & $374(100)$ & $678(100)$ & $1052(100)$ & $228(17.8)$ \\
\hline \multicolumn{5}{|c|}{ Alcohol consumption in g/day according to the WHO drinking categoriesł } \\
\hline Abstainers & $172(53.9)$ & $152(24.7)$ & $324(34.7)$ & \\
\hline $\mathrm{DI}$ & $135(42.3)$ & $430(69.8)$ & $565(60.4)$ & \\
\hline DII & $11(3.4)$ & $23(3.7)$ & $34(3.6)$ & \\
\hline DIII & $1(0.3)$ & $11(1.8)$ & $12(1.3)$ & \\
\hline $\mathrm{N}(\%)$ & $319(100)$ & $616(100)$ & $935(100)$ & $283(22.1)$ \\
\hline \multicolumn{5}{|l|}{ Body mass index } \\
\hline BMI $<25$ (underweight-normal) & $125(27.4)$ & $151(19.3)$ & $276(22.3)$ & \\
\hline BMI 25.00-29.99 (overweight/pre-obese) & $191(41.8)$ & $401(51.3)$ & $592(47.8)$ & \\
\hline BMI 30.00-39.99 (obese class I+II) & $132(28.9)$ & $212(27.1)$ & $344(27.8)$ & \\
\hline $\mathrm{BMI} \geq 40$ (obese class III) & $9(2)$ & $18(2.3)$ & $27(2.2)$ & \\
\hline $\mathrm{N}(\%)$ & $457(100)$ & $782(100)$ & $1239(100)$ & $41(3.2)$ \\
\hline \multicolumn{5}{|c|}{ Chronic care services utilisation (physician-reported) } \\
\hline \multicolumn{5}{|l|}{ Enrolment in DMP-CHD } \\
\hline No & $372(77.8)$ & $521(65)$ & $893(69.8)$ & \\
\hline Yes & $106(22.2)$ & $281(35)$ & $387(30.2)$ & \\
\hline N (\%) & $478(100)$ & $802(100)$ & $1280(100)$ & $0(0)$ \\
\hline \multicolumn{5}{|l|}{ Duration of enrolment in DMP-CHD } \\
\hline Low (0.5-3 years) & $61(64.2)$ & $154(60.4)$ & $215(61.4)$ & \\
\hline High (4-7 years) & $34(35.8)$ & $101(39.6)$ & $135(38.6)$ & \\
\hline $\mathrm{N}(\%)$ & $95(100)$ & $255(100)$ & $350(100)$ & 37 (9.6) \\
\hline
\end{tabular}


Table 2 Continued

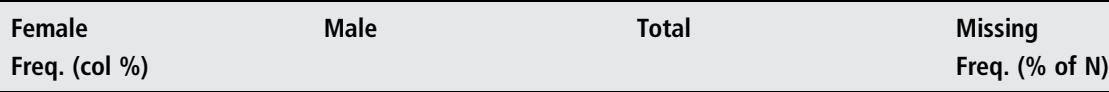

Social situation

Living in a partnership

Yes $\quad 178(51.7) \quad 528(85.3)$

$\mathrm{N}(\%) \quad 344(100) \quad 619(100) \quad 963(100)$

$317(24.8)$

Social contacts (family members/friends whom participants can count on/discuss problems with)

$0-1$

2-4

5-10 and more

$\mathrm{N}(\%)$

Current economic activity

Full-time employment

Minor/occasional employment

Part-time employment

Housewife/domestic activity

Retired

Unemployed

$\mathrm{N}(\%)$

Practice-level characteristics

Gender of primary care physician

Male

Female

$\mathrm{N}(\%)$

Area characteristics

Regional deprivation of patients' residential areas (GIMD)

T1 (least deprived)

$\mathrm{T} 2$

T3 (most deprived)

$\mathrm{N}(\%)$

Degree of urbanisation

Rural

Urban cluster

High-density cluster

$\mathrm{N}(\%)$

$$
\begin{gathered}
48(14.2) \\
184(54.4) \\
106(31.4) \\
338(100) \\
3(0.8) \\
4(1.1) \\
5(1.4) \\
129(35.4) \\
222(61) \\
1(0.3) \\
364(100)
\end{gathered}
$$

$332(73.6)$

119 (26.4)

$451(100)$

57 (12)

178 (37.5)

240 (50.5)

$475(100)$

$99(20.8)$

$288(60.6)$

88 (18.5)

$475(100)$
93 (14.7)

331 (52.2)

210 (33.1)

634 (100)

32 (4.8)

$4(0.6)$

$5(0.7)$

$4(0.6)$

$620(92.1)$

8 (1.2)

673 (100)
141 (14.5)

515 (53)

316 (32.5)

972 (100)

308 (24.1)

35 (3.4)

$8(0.8)$

10 (1)

133 (12.8)

842 (81.2)

$9(0.9)$

1037 (100)

243 (19.0)

588 (76.9)

920 (75.7)

296 (24.3)

1216 (100)

$64(5.0)$

765 (100)

120 (15.2)

177 (14)

484 (38.2)

605 (47.8)

1266 (100)

$14(1.1)$

365 (46.1)

791 (100)

274 (21.6)

775 (61.2)

217 (17.1)

1266 (100)

All data refer to the 8-year follow-up phase (t3: 2008-2010) if not otherwise stated.

*Highest educational attainment: Level I: no degree or minimum of 9 years of education qualifying for professional training (Hauptschule). Level II: minimum of 10-11 years of education qualifying for professional training (Realschule/Mittlere Reife). Level III: minimum of 12-13 years of education qualifying for university entrance (Fachhochschulreife/Abitur). ¥Alcohol consumption in g/day: DI: female: 0-19.99; male: 0-39.99. DII: female: 20-39.99; male: 40-59.99. DIII: female: $>40.0 ;$ male: $>60.0$. T1-T3: Tertiles of the German Index of

Multiple Deprivation (GIMD).

Col\%, column percent; Freq., absolute frequency; M, arithmetic Mean.

indicated a social gradient, but these were not statistically significant as judged by the $95 \%$ CIs (table 3 ).

Models adjusted for individual-level factors and higher level variables

Morbidity-adjusted models—-fixed effects

The trends observed in the crude analysis for the effects of educational attainment on DMP enrolment (table 3) were attenuated when adjusting for the severity of comorbidities (CIRS-G), age and sex (table 4, M1). This indicates an equal enrolment in the DMP for equal need (measured by CIRS-G) between patients with different educational attainments.

Additional adjustment for practice-level factors did not change the relationship between educational attainment and DMP enrolment (table 4, M2). The trends observed in the crude analysis for the effects of regional deprivation on DMP enrolment (table 3) were attenuated in the model which adjusted for differences in comorbidities, age, sex, educational attainment and gender of GPs (table 4, M3).
Additional adjustment for differences in the degree of urbanisation further attenuated the deprivation effects in the comorbidity model (table 4, M4). Compared to rural areas, the odds of enrolment in the DMP was $44 \%$ lower $(\mathrm{OR}=0.56$ $(0.31$ to 1.01$))$ in high-density clusters, and $17 \%$ lower $(\mathrm{OR}=0.83(0.55$ to 1.25$))$ in urban clusters, adjusted for the other covariables in the model (table 4, M4).

Male patients had significantly higher odds of being enrolled in the DMP-CHD in crude (table 3 ) and all comorbidity-adjusted models (table 4, M1-4). In the fully adjusted model, the odds for enrolment to the DMP-CHD among men were 2.16 (1.57 to 2.98) times the odds of women adjusted for age, severity of comorbidities, educational attainment and contextual effects at practice-level and municipality-level (table 4, M4).

Morbidity-adjusted models—random effects

The variance in DMP enrolment between practices $(\mathrm{MOR}=1.74)$ was larger than the between-municipality variance $(\mathrm{MOR}=1.30)$ in the unconditional model (table $4, \mathrm{M} 0)$. 
Table 3 Crude ORs for the association between enrolment in the Disease Management Programme for coronary heart diseases and individual-level, practice-level and area-level variables obtained from cross-classified multilevel logistic regression models

\begin{tabular}{|c|c|c|c|c|}
\hline & Crude OR & $95 \% \mathrm{Cl}$ & $\mathrm{N}^{*}$ & Practice $\mathrm{N}$ \\
\hline \multicolumn{5}{|l|}{ Individual-level exposure } \\
\hline \multicolumn{5}{|l|}{ Education (Ref. I, lowest) } \\
\hline II & 0.98 & (0.65 to 1.48$)$ & 1241 & 382 \\
\hline III (highest) & 1.45 & (0.97 to 2.15 ) & & \\
\hline \multicolumn{5}{|l|}{ Sociodemographics } \\
\hline \multicolumn{5}{|l|}{ Age group (Ref: 55-64) } \\
\hline $65-74$ & 1.33 & (0.88 to 1.99 ) & 1241 & 382 \\
\hline $75-84$ & 1.04 & (0.68 to 1.59 ) & & \\
\hline Male (Ref: female) & 1.98 & (1.50 to 2.62 ) & 1241 & 382 \\
\hline \multicolumn{5}{|l|}{ Behavioural risk factors } \\
\hline Hypercholesterolaemia (yes vs no) & 1.20 & (0.89 to 1.60$)$ & 1160 & 368 \\
\hline \multicolumn{5}{|l|}{ Body mass index (Ref: normal/underweight) } \\
\hline Overweight/preobese & 1.11 & (0.80 to 1.55$)$ & 1202 & 378 \\
\hline Obese class I+II & 0.91 & (0.62 to 1.32$)$ & & \\
\hline Obese class III & 1.10 & (0.44 to 2.79 ) & & \\
\hline \multicolumn{5}{|l|}{ Smoking status (Ref: never smoker) } \\
\hline Former & 1.29 & (0.97 to 1.73 ) & 1037 & 358 \\
\hline Current & 1.15 & (0.68 to 1.94$)$ & & \\
\hline \multicolumn{5}{|l|}{ Alcohol consumption (Ref: abstainers) $\dagger$} \\
\hline $\mathrm{DI}$ & 1.58 & (1.15 to 2.17 ) & 920 & 335 \\
\hline DII & 1.18 & (0.53 to 2.66$)$ & & \\
\hline DIII & 1.88 & (0.52 to 6.76$)$ & & \\
\hline \multicolumn{5}{|l|}{ Comorbidities } \\
\hline Cumulative Illness Rating Scale for Geriatrics—severity indexł & 1.45 & (1.05 to 2.00$)$ & 1032 & 334 \\
\hline \multicolumn{5}{|l|}{ Ethnicity } \\
\hline Immigration background (yes vs no) & 1.37 & (0.86 to 2.18$)$ & 1234 & 381 \\
\hline \multicolumn{5}{|l|}{ Social situation } \\
\hline Certified and approved need of long-term care (yes vs no) & 0.49 & (0.21 to 1.13 ) & 964 & 346 \\
\hline Living in a partnership (yes vs no) & 1.20 & (0.86 to 1.68$)$ & 949 & 347 \\
\hline \multicolumn{5}{|l|}{ Social contacts (Ref.: 0-1 contacts) } \\
\hline $2-4$ & 1.36 & (0.87 to 2.11$)$ & 958 & 345 \\
\hline $5-10$ and more & 1.15 & (0.72 to 1.84$)$ & & \\
\hline \multicolumn{5}{|l|}{ Practice-level variables } \\
\hline Male general practitioner (vs female) & 0.97 & (0.68 to 1.40$)$ & 1204 & 365 \\
\hline \multicolumn{5}{|l|}{ Area-level variables } \\
\hline \multicolumn{5}{|l|}{ Regional deprivation (Ref.: T1, lowest deprivation)§ } \\
\hline $\mathrm{T} 2$ & 0.62 & (0.37 to 1.04$)$ & 1241 & 382 \\
\hline T3 (highest deprivation) & 0.74 & (0.44 to 1.24$)$ & & \\
\hline \multicolumn{5}{|l|}{ Degree of urbanisation (Ref: rural) } \\
\hline Urban cluster & 0.90 & (0.59 to 1.35$)$ & 1241 & 382 \\
\hline High-density cluster & 0.60 & (0.28 to 1.30$)$ & & \\
\hline
\end{tabular}

Individual-level variables (age, sex, educational attainment) and the severity of comorbidities explained $12.5 \%$ of the betweenmunicipality variance. Differences in individual-level variables could thus not fully explain the between-municipality variance in DMP enrolment. Regional deprivation explained $28.6 \%$ of the residual between-municipality variance $(\mathrm{MOR}=1.29)$ that remained after full adjustment for individual-level variables (table 4, M2). Further adjustment for the degree of urbanisation reduced the residual between-municipality variance by another $71.4 \%$, so that $100 \%$ of the (comparably small) between- municipality variance was explained by regional deprivation and the degree of urbanisation (table 4, M4).

\section{Models adjusted for individual-level behavioural risk factors} and the social situation

Adjusting the relationship between educational attainment and DMP enrolment for behavioural risk factors and the social situation (see online supplementary file 1, tables S1-S2) attenuated the trends observed in the crude analysis and confirmed the results of the comorbidity-adjusted models. Regional 
Table 4 Effect estimates of individual-level comorbidity, practice-level and area-level variables on the enrolment in the Disease Management Programme for coronary heart disease obtained from cross-classified multilevel logistic regression models

Measures of association/fixed effects-OR $(95 \% \mathrm{Cl})$

$\begin{array}{llllll}\text { M0 } & \text { M1 } & \text { M2 } & \text { M3 } & \text { M4 }\end{array}$

Individual-level variables

Education (Ref. I, lowest)

III (highest)

1.14

1.14

$(0.71$ to 1.82

(0.75 to 1.82$)$

1.17

(0.76 to 1.84$)$

1.18

Sociodemographics

Age group (Ref: 55-64)

65-74

75-84

Male (vs female)

(0.91 to 2.25$) \quad 1.43$

Comorbidities

CIRS-G—severity index*

(0.73 to 1.88 )

(0.91 to 2.25$)$

(0.73 to 1.88$)$

(1.59 to 3.01 )

1.44

(0.92 to 2.27 )

(0.74 to 1.89 )

(1.58 to 2.98 )

1.46

(1.59 to 3.01 ) 2.19

(1.09 to 2.11 )

1.51

(1.08 to 2.10$)$

1.52

Contextual variables

Practice-level

Male general practitioner (vs female)

(0.66 to 1.47$)$

(0.68 to 1.54$)$

Area level

Regional deprivation (Ref.:T1-least deprived)

T3 (most deprived)

Degree of urbanisation (Ref: rural)

Urban cluster

High-density cluster

Intercept

Measures of variation/random effects

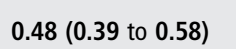

0.11

(0.05 to 0.24)

0.11

(0.05 to 0.24 )

0.68

(0.41 to 1.15$)$

0.70

(0.48 to 1.35 )

0.92

(0.42 to 1.14$)$

Practice-variance

MOR-Practice

0.32

0.33

1.74

1.72

PCV (\%) Practice

$\begin{array}{ll}1.74 & -3.0^{+} \\ - & 0.08\end{array}$

Municipality-variance

MOR-Municipality

PCV (\%) Municipality

Model fit and sample size

Wald- $\chi^{2}$ (df)

Model-sig. ( $p$ value)

0.08

1.3

0.07

1.29

$-12.5^{+}$

$14.87(2) \ddagger \quad 33.17(6)$

$0.0006<0.0001$

Practice $\mathrm{N}$

1000

1000

319

0.32
1.72
$0.0^{++}$
0.07
1.29
$0.0^{++}$

$33.18(7)$
$<0.0001$
1000
319

0.14

(0.06 to 0.33 )

0.83

0.56
0.15

(0.55 to 1.25$)$

(0.31 to 1.01 )

(0.06 to 0.37 )

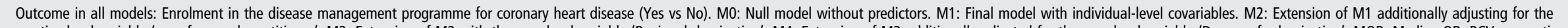

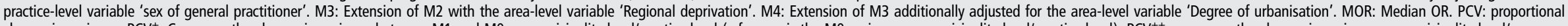
change in variance. $\mathrm{PCV}^{+}$: Compares the change in variance between M1 and M0 on municipality-level/practice-level (reference is the M0 variance on municipality-level/practice-level). PCV practice-level between the models with contextual variables (M2-M4) and the final model containing individual variables (M1), respectively (reference is the M1 variance on municipality-level/practice-level).

${ }^{*}$ Cumulative Illness Rating Scale (CIRS)—-severity index: The OR shows the effect on the propensity of enrolment of a one unit difference in CIRS-severity index, comparing patients with a higher index with patients with a lower index.

tRegional deprivation tertiles (T1-T3) refer to the deprivation of the patient-residential area.

₹Test statistic of a likelihood-ratio test, testing the fit of the null model against a single-level logistic regression model.

Bold figures indicate ORs that are significantly different from 1 .

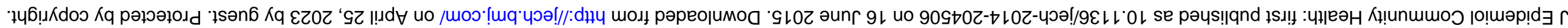


deprivation of the patient-residential area was significantly negatively associated with the odds of DMP enrolment when comparing municipalities with medium deprivation (T2) with the least deprived municipalities (T1) regardless of differences in individual educational attainment, age, sex, individual behavioural risk factors, social situation, migration background, practice-level characteristics and the degree of urbanisation (see online supplementary file 1 , tables S1-S2). The association was negative, but in all models not significant when comparing most deprived with least deprived municipalities. The relationship between regional deprivation and DMP enrolment was partly explained by differences in the severity of comorbidities (CIRS-G; table 4, M3).

The strength of association between male gender and DMP enrolment in fully adjusted models (see online supplementary file 1, table S1-S2) was smaller compared to the effects observed in the comorbidity-adjusted model (table 4), but significant and consistent in direction $(\mathrm{OR}=1.60$ (1.08 to 2.36) adjusted for individual-level behavioural risk factors, age, sex, educational attainment, practice-level and municipality-level variables (see online supplementary file 1 , table $\mathrm{S} 1$ ) and $\mathrm{OR}=1.79$ (1.23 to 2.61) adjusted for the social situation, age, sex, educational attainment, practice-level and municipality-level variables (see online supplementary file 1 , table S2)). Not considering differences in comorbidities thus underestimated the effects of gender on DMP enrolment.

Details on the model specification and regression diagnostics are provided in online supplementary file 1 .

\section{DISCUSSION}

This is the first study to analyse social disparities in DMP enrolment among elderly people with CHD in Germany, taking into account both a wide range of individual-level factors and contextual effects at the levels of municipalities and primary care practices. We found that female gender and higher regional deprivation at a small-area level (comparing medium with least-deprived municipalities) are negatively associated with enrolment in the DMP-CHD, regardless of individual-level factors and potential confounding by variables at practice-level (physician gender) and/or area-level (degree of urbanisation). Adjustment for differences in severity of comorbidities, however, attenuated the effects of regional deprivation on DMP enrolment.

Our findings provide evidence that the health system allowed marked gender disparities in DMP enrolment that have not been reported as yet. ${ }^{25}$ Routine evaluations across sickness funds in a large federal state show an imbalanced gender distribution (63\% male among approximate 228000 DMP-CHD participants). ${ }^{27}$ These routine DMP evaluation reports, which build on claims data of the SHI, only report the gender distribution within DMP participants. As there are no data on nonparticipants (which would be a natural control group), it is not possible to quantify disparities against an external standard. Routine state-wide evaluations in Saarland do not report sexstratified data on the approximate 22100 patients (mean age: 70 years) enrolled in the DMP-CHD in $2010,{ }^{28}$ hampering comparisons with our sample. General claims data of the SHI allow evaluation of the gender distribution between DMP-participants and non-participants among their members, but analysis of disparities are limited by lacking information on individual covariables including SES indicators.

We have shown that the differences in the individual propensity of enrolment in the DMP-CHD between male and female patients could neither be explained by differences in age, individual SES, lifestyle, severity of comorbidity (CIRS-G) and the social situation, nor by differences attributable to physicians' gender and area-level characteristics. The phenomenon of gender differences in prevalence, manifestation, diagnosis, treatment and prognosis of CHD is well known and is often attributed to stereotypes among physicians about the disease as afflicting primarily men. ${ }^{29}$

We found that regional deprivation at a small-area level, comparing medium with least deprived municipalities, is independently associated with enrolment in the DMP-CHD, regardless of the composition of municipalities as far as patient-level factors such as age, sex, educational attainment, behavioural risk factors and social situation/migration (see online supplementary file 1, table S1-S2) are concerned. This effect was not confounded by variables at practice-level (physician gender) and/or area-level (degree of urbanisation). Compositional effects existed as far as comorbidity profiles are concerned. Adjusting for the CIRS-G severity index attenuated the statistical significance of the GIMD effects, while the point estimates still indicated a negative association with DMP enrolment. Additional adjustment for the degree of urbanisation further attenuated the deprivation effects, but a trend indicating a negative association remained.

Contextual disparities in utilisation of DMPs may arise through: differences in (1) enabling municipality resources, ${ }^{11} 14$ (2) supply of healthcare services, ${ }^{11} 14$ (3) different direct and indirect costs ${ }^{30}$ for patients in attending the quarterly GP visits entailed in DMP enrolment, and (4) different perceptions of perceived benefit ${ }^{30}$ of enrolment in DMPs depending on the residential environment. Evidence from aggregate data analysis suggests that supply-side determinants indeed have a relevant impact on equity of outpatient care in Germany, including the Saarland. ${ }^{31}$ Previous studies among the ESTHER cohort have provided evidence for inequities in out-of-pocket payments among elderly patients when utilising chronic care services. ${ }^{32}$ The question whether and how regional deprivation affects the degree to which 'potential access' to the DMP-CHD (guaranteed by the SHI system) is converted into 'realised access ${ }^{33}$ deserves further investigation.

The non-linear relationship between regional deprivation of patient-residential areas with DMP enrolment deserves further investigation, ideally in settings with more clusters (ie, municipalities). The odds for DMP enrolment for most-deprived compared to least-deprived areas were consistently not as low as those for medium-deprived compared to least-deprived areas. Assuming a social gradient related to deprivation, one would expect the enrolment in most-deprived municipalities to be lower than that in medium-deprived compared to the leastdeprived municipalities. Barriers may exist in medium-deprived municipalities that are not present in most-deprived municipalities: they may interact with access to DMP enrolment, but remained unmeasured in our study.

We found no evidence for disparities based on the highest educational attainment, despite existing mechanisms which (theoretically) have the potential to create disparities in DMP enrolment. In this respect, access to (or rather utilisation of) the DMP-CHD is equitably distributed. This finding is consistent with two studies that assessed the possibility of selective enrolment in enrolment in DMP-CHD using self-reported ${ }^{26}$ and physician-reported ${ }^{25}$ enrolment status. It affirms that differences in individual educational attainment do not constitute a barrier in uptake of the DMP-CHD. Only one study focusing on DMPs for diabetes type II found significant differences in educational attainment between DMP participants and non-participants using nationwide claims data of a large sickness fund. ${ }^{34}$ None of the other previous studies on DMPs in Germany were able to 
assess the possibility of social selection processes between participants and non-participants due to the limited availability of information on SES and/or lack of control groups. ${ }^{8} 93536$

We found a substantial variation in the odds of enrolment between municipalities in unconditional models (range: MOR: 1.30-1.56). In fully adjusted models, the complete residual between-municipality variance (that remained after controlling for individual-level variables) could be explained by differences in two variables: regional deprivation and the degree of urbanisation. This means that small-area variation in enrolment to the programme is to a large extent explained by differences in regional deprivation and degree of urbanisation. This marks out the small-area context as an important factor to be taken into account in future studies on programme effectiveness and its impact on equity and efficiency.

\section{STRENGTHS AND LIMITATIONS}

The major strength of our study is that we considered a wide range of individual-level factors in assessing disparities in DMPs, while taking into account that patients are simultaneously clustered in practices and municipalities. This minimised the possibility of biased SEs and inferences, ${ }^{37}$ although the number of municipalities was comparably small with large differences in population sizes. Since the number of higher level clusters is the most important criterion for assessing fixed effects of higher level variables, ${ }^{38}$ our study is likely to be underpowered as far as the effects of regional deprivation are concerned. This might explain why the comparison 'highest versus lowest deprivation' was not statistically significant, despite the fact that the point estimates (GIMD T3 vs T1) were consistently smaller than 1 in all models, indicating a trend towards a potentially relevant negative association.

In analysing disparities, we adjusted for differences in risk factors and comorbidities ${ }^{21}$ to ensure that potential socioeconomic differences in DMP enrolment are corrected for underlying medical needs. In contrast to models adjusting for risk factors, adjustment for the severity of comorbidities attenuated the significance of the deprivation effects. This demonstrates the need for further studies with a larger number of municipalities. Although we considered medical need, we cannot completely rule out that the regional or gender disparities are not (at least partially) due to individual preferences, because no variables captured these tendencies.

As part of a covariable selection process, we built three separate models with different sets of confounders that were considered because of their (hypothesised) relevance to the potential selection process in DMP enrolment. At the end, however, we did not include all covariables in one model, for example, risk factors and social factors together into the model controlling for medical need. The reasons for that are provided in online supplementary file 1 .

Our findings are limited by the possibility of attrition bias: no information on DMP status was available for participants who dropped out or died before 2008, since this was not the primary outcome of the cohort study. Compared to the cohort population at $\mathrm{t} 3(\mathrm{~N}=7012)$, the proportion of individuals with lowest educational attainment was significantly higher (about 7 percentage-points) among the individuals $(\mathrm{N}=2937)$ who were a priori excluded (see online supplementary file 2). At baseline, those excluded were significantly older and more likely to be retired or pursue domestic activities. The prevalence of selfreported CHD was also significantly higher (about 5 percentage points) among those excluded. Therefore, the finding that educational attainment was not associated with DMP enrolment might be an underestimate of the true effect operating at the individual level. Further details on the non-responder analysis are reported in online supplementary file 2 .

Also, GPs reported only about the current enrolment status and no information was available on whether individuals categorised as 'not enrolled' used to be enrolled but dropped out until t3. Furthermore, we did not explicitly address the possibility of cohort effects ${ }^{15}$ as far as the effect of education as a measure of SES is concerned, since the meaning of educational attainment varies for different birth cohorts. ${ }^{39}$ The majority of our sample was retired or pursued domestic activities. Thus, measuring individual-level SES with wealth-based indicators or measures that reflect household conditions for this age group would have better reflected individual $\mathrm{SES}^{39}$ and could have thus yielded different results. However, these data were not captured, so the analysis made use of the best available indicators.

Finally, the small average number of patients with CHD per a higher level unit may have limited our aim to comprehensively assess random effects. ${ }^{38}$ However, the underlying model assumptions (see online supplementary file 1) regarding the residual variance at a higher level were sufficiently met despite the small average cluster sizes.

\section{CONCLUSION}

The individual propensity for enrolment in DMP-CHD among elderly patients with CHD was markedly higher for men compared to women after full adjustment for individual-level, practice-level and area-level variables. Access to or utilisation of

\section{What is already known on this subject}

- Disease Management Programmes for patients with coronary heart disease in Germany aim to improve health outcomes and quality of care in terms of stronger guideline-orientation.

- Systematic selection effects in programme enrolment have been found depending on patients' age and (co)morbidity.

- No studies have as yet analysed social disparities in a control-group design taking into account both individual and contextual factors.

\section{What this study adds}

- Enrolment in the Disease Management Programme for coronary heart disease suffers from gender disparities, and there is a trend towards small-area disparities, after controlling for possible individual and area-level confounders.

- Small-area variation in enrolment to the programme is to a large extent explained by differences in regional deprivation and degree of urbanisation which marks out the small-area context as an important factor to be taken into account in future studies.

- Individual educational attainment did not constitute a barrier towards uptake of the programme, regardless of differences in behavioural risk factors, comorbidity, social situation, place of residence or gender of patients' general practitioners. 
the DMP-CHD was thus inequitably distributed across gender. GPs offering DMPs for patients with CHD should be aware of potential gender stereotypes in their daily practice. No disparities were observed across individual-level educational attainment indicating that the utilisation of the DMP-CHD is equitably distributed in this respect, although this finding needs to be interpreted in the light of the possibility of attrition bias. Regional deprivation was negatively associated with DMP enrolment independent of individual educational attainment and behavioural/social factors. Adjustment for differences in comorbidities attenuated the deprivation effects, but a trend indicating disparities remained which deserves further investigation. Future research on DMPs should consider the small-area context as a relevant factor in its own right.

\section{Author affiliations \\ 'Department of General Practice and Health Services Research, University Hospital Heidelberg, Heidelberg, Germany \\ ${ }^{2}$ Institute of Health Economics and Health Care Management, Helmholtz Zentrum München-German Research Center for Environmental Health $(\mathrm{GmbH})$, Neuherberg, Germany \\ ${ }^{3}$ Division of Clinical Epidemiology and Aging Research, German Cancer Research Center (DKFZ), Heidelberg, Germany \\ ${ }^{4}$ Institute of Medical Biometry and Informatics, University Heidelberg, Heidelberg, Germany \\ ${ }^{5}$ Saarland Cancer Registry, Saarbrücken, Germany \\ ${ }^{6}$ Department of Epidemiology and International Public Health, School of Public Health, Bielefeld University, Bielefeld, Germany}

Acknowledgements The authors would like to thank the ESTHER study participants, the study physicians and the ESTHER study office in the state of Saarland, as well as the general practitioners of the state of Saarland for their very close and long-standing cooperation. They are also grateful for the valuable comments of Jürgen Breckenkamp and Odile Sauzet (both: Department of Epidemiology and International Public Health, School of Public Health, Bielefeld University) on the preliminary results of this study.

Contributors KB conceived the study, analysed the data and wrote the manuscript. WM took part in data collection and analysis of the GIMD and critical revision of the manuscript. HB conceived the cohort study, data collection and critical revision of the manuscript. K-US was involved in data collection and analysis and critical revision of the manuscript. CS gave statistical advice, was involved in important contribution to data interpretation and critical revision of manuscript. AM made important contribution to the study design and critical revision of the manuscript. BH participated in data collection and critical revision of the manuscript. JS contributed to the study design critical and revision of the manuscript. OR made important contribution to the study design and critical revision of the manuscript.

Funding The study was funded by the young scientists programme of the German network 'Health Services Research Baden-Württemberg' of the Ministry of Science, Research and Arts in collaboration with the Ministry of Employment and Social Order, Family, Women and Senior Citizens, Baden-Württemberg. The ESTHER study is supported by the Baden-Württemberg State Ministry of Science, Research and Arts and by the German Federal Ministry of Education and Research (grant no. 01ET0717).

Competing interests None declared.

Patient consent Obtained.

Ethics approval The ESTHER study was approved by the ethics committees of the medical faculty of the University of Heidelberg and of the medical board of the state of Saarland.

Provenance and peer review Not commissioned; externally peer reviewed.

Open Access This is an Open Access article distributed in accordance with the Creative Commons Attribution Non Commercial (CC BY-NC 4.0) license, which permits others to distribute, remix, adapt, build upon this work non-commercially, and license their derivative works on different terms, provided the original work is properly cited and the use is non-commercial. See: http://creativecommons.org/ licenses/by-nc/4.0/

\section{REFERENCES}

1 Murray CJ, Vos T, Lozano R, et al. Disability-adjusted life years (DALYs) for 291 diseases and injuries in 21 regions, 1990-2010: a systematic analysis for the Global Burden of Disease Study 2010. Lancet 2012;380:2197-223.
2 Lozano R, Naghavi M, Foreman K, et al. Global and regional mortality from 235 causes of death for 20 age groups in 1990 and 2010: a systematic analysis for the Global Burden of Disease Study 2010. Lancet 2012;380:2095-128.

3 Federal Statistical Office (Destatis). [Todesursachen in Deutschland]. http://www destatis de Fachserie 12, Reihe 4, 2014. https://www.destatis.de/DE/Publikationen/ Thematisch/Gesundheit/Todesursachen/Todesursachen2120400127004.pdf? blob=publicationFile (accessed 1 Apr 2014).

4 Szecsenyi J, Rosemann $T$, Joos S, et al. German diabetes disease management programs are appropriate for restructuring care according to the chronic care model: an evaluation with the patient assessment of chronic illness care instrument. Diabetes Care 2008;31:1150-4.

5 Busse R. Disease management programs in Germany's statutory health insurance system. Health Affair 2004;23:56-67.

6 Joint Federal Commission (G-BA). [Zwanzigste Verordnung zur Änderung der Risikostruktur-Ausgleichsverordnung (20.RSA-ÄndV) Vom 23. Juni 2009]. Bundesgesetzblatt 2009;1:1551-61.

7 German Federal (Social) Insurance Office (BVA). [Zulassung der Disease Management Programme (DMP) durch das Bundesversicherungsamt (BVA)]. 2014 http://www bundesversicherungsamt de; http://www.bundesversicherungsamt.de/ weitere-themen/disease-management-programme/zulassung-disease-managementprogramme-dmp.html\#c207 (accessed 1 Apr 2014).

8 Drabik A, Sawicki PT, Muller D, et al. [The methods within the evaluation of disease management programmes in control-group designs using the example of diabetes mellitus - a systematic literature review]. Gesundheitswesen 2012;74:496-501.

9 Schäfer I, Küver C, Gedrose B, et al. Selection effects may account for better outcomes of the German Disease Management Program for type 2 diabetes. BMC Health Serv Res 2010:10:351.

10 Aday LA, Andersen R. A framework for the study of access to medical care. Health Serv Res 1974:9:208-20.

11 Chaix B. Geographic life environments and coronary heart disease: a literature review, theoretical contributions, methodological updates, and a research agenda. Annu Rev Public Health 2009:30:81-105.

12 Kirby JB, Kaneda T. Neighborhood Socioeconomic Disadvantage and Access to Health Care. J Health Soc Behav 2005:46:15-31.

13 Diez Roux AV, Mair C. Neighborhoods and health. Ann NY Acad Sci 2010;1186:125-45

14 Phillips KA, Morrison KR, Andersen R, et al. Understanding the context of healthcare utilization: assessing environmental and provider-related variables in the behavioral model of utilization. Health Serv Res 1998:33(3 Pt 1):571-96.

15 Raum E, Rothenbacher D, Low M, et al. Changes of cardiovascular risk factors and their implications in subsequent birth cohorts of older adults in Germany: a life course approach. Eur J Cardiovasc Prev Rehabil 2007;14:809-14.

16 Maatouk I, Wild B, Wesche D, et al. Temporal predictors of health-related quality of life in elderly people with diabetes: results of a German cohort study. PLOS ONE 2012; 7:e31088

17 Schöttker B, Haug U, Schomburg L, et al. Strong associations of 25-hydroxyvitamin $D$ concentrations with all-cause, cardiovascular, cancer, and respiratory disease mortality in a large cohort study. Am J Clin Nutr 2013;97:782-93.

18 Maier $W$, Holle $\mathrm{R}$, Hunger $\mathrm{M}$, et al. The impact of regional deprivation and individual socio-economic status on the prevalence of Type 2 diabetes in Germany. A pooled analysis of five population-based studies. Diabet Med 2013;30:e78-86.

19 Maier W, Fairburn J, Mielck A. [Regional deprivation and mortality in Bavaria. Development of a community-based index of multiple deprivation] Gesundheitswesen 2012;74:416-25

20 Miller MD, Paradis CF, Houck PR, et al. Rating chronic medical illness burden in geropsychiatric practice and research: application of the Cumulative Illness Rating Scale. Psychiat Res 1992;41:237-48.

21 Le Cook B, McGuire TG, Zaslavsky AM. Measuring racial/ethnic disparities in health care: methods and practical issues. Health Serv Res 2012;47:1232-54.

22 European Commission. Glossary: Revision of the degree of urbanisation. 2014. http://epp eurostat ec europa eu/statistics_explained/index php/Glossary:Degree_of urbanisation; http://epp.eurostat.ec.europa.eu (accessed 25 Mar 2014).

23 Merlo J, Chaix B, Ohlsson $\mathrm{H}$, et al. A brief conceptual tutorial of multilevel analysis in social epidemiology: using measures of clustering in multilevel logistic regression to investigate contextual phenomena. J Epidemiol Community Health 2006:60:290-7.

24 Merlo J, Yang M, Chaix B, et al. A brief conceptual tutorial on multilevel analysis in social epidemiology: investigating contextual phenomena in different groups of people. J Epidemiol Community Health 2005;59:729-36.

25 Stark R, Kirchberger I, Hunger $M$, et al. Improving care of post-infarct patients: effects of disease management programmes and care according to international guidelines. Clin Res Cardiol 2014;103:237-45.

26 Gapp 0, Schweikert B, Meisinger C, et al. Disease management programmes for patients with coronary heart disease-an empirical study of German programmes. Health Policy 2008:88:176-85.

27 Hagen B, Altenhofen L, Groos S, et al. Qualitätssicherungbericht 2012: disease management programme in Nordrhein. Nordrheinische Gemeinsame Einrichtung Disease-Management Programme GbR, 2013 
28 Gemeinsame Einrichtung Saarland. Indication-specific report of the joint commission for the DMP Coronary Heart Disease: report for the period 2010/07/01-2010/ 12/31. Gemeinsame Einrichtung Saarland—Kassenärztliche Vereinigung Saarland, 2011. 1-5-2014.

29 Swahn $\mathrm{E}$. The care of patients with ischaemic heart disease from a gender perspective. Eur Heart J 1998;19:1758-65.

30 Goddard M, Smith P. Equity of access to health care services: theory and evidence from the UK. Soc Sci Med 2001;53:1149-62.

31 Ozegowski S, Sundmacher L. Understanding the gap between need and utilization in outpatient care - the effect of supply-side determinants on regional inequities. Health Policy 2014;114:54-63.

32 Bock JO, Matschinger $\mathrm{H}$, Brenner $\mathrm{H}$, et al. Inequalities in out-of-pocket payments for health care services among elderly Germans-results of a population-based cross-sectional study. Int I Equity Health 2014;13:3.

33 Aday LA, Andersen R. Equity of access to medical care: a conceptual and empirical overview. Med Care 1981;19:4-27.
34 Elkeles T, Kirschner W, Graf C, et al. Health care in and outside a DMP for type 2 diabetes mellitus in Germany-results of an insurance customer survey focussing on differences in general education status. J Public Health 2009;17:205-16.

35 Miksch A, Laux G, Ose D, et al. Is there a survival benefit within a German primary care-based disease management program? Am J Manag Care 2010;16:49-54.

36 Bestehorn K, Jannowitz C, Karmann B, et al. Characteristics, management and attainment of lipid target levels in diabetic and cardiac patients enrolled in Disease Management Program versus those in routine care: LUTZ registry. BMC Public Health 2009;9:280.

37 Pickett KE, Pearl M. Multilevel analyses of neighbourhood socioeconomic context and health outcomes: a critical review. J Epidemiol Community Health 2001;55:111-22.

38 Snijders T. Power and sample size in multilevel linear models. In: Everitt B, Howell D, eds. Encylopedia of statistics in behavioural science. Chicester: Wiley, 2005:1570-3.

39 Galobardes B, Shaw M, Lawlor DA, et al. Indicators of socioeconomic position (part 1). J Epidemiol Community Health 2006;60:7-12. 